\title{
DYNAMICAL STRUCTURE OF THE MOLECULAR INTERSTELLAR MEDIUM IN AN EXTREMELY BRIGHT, MULTIPLY LENSED $z \simeq 3$ SUBMILLIMETER GALAXY DISCOVERED WITH HERSCHEL
}

\author{
Dominik A. Riechers ${ }^{1,28}$, A. Cooray ${ }^{1,2}$, A. OMOnT ${ }^{3}$, R. Neri ${ }^{4}$, A. I. Harris ${ }^{5}$, A. J. Baker ${ }^{6}$, P. Cox ${ }^{4}$, D. T. FraYer ${ }^{7}$, \\ J. M. Carpenter ${ }^{1}$, R. Auld ${ }^{8}$, H. Aussel ${ }^{9}$, A. Beelen ${ }^{10}$, R. Blundell ${ }^{11}$, J. Bock ${ }^{1,12}$, D. Brisbin ${ }^{13}$, D. Burgarella ${ }^{14}$, \\ P. Chanial $^{9}$, S. C. Chapman ${ }^{15}$, D. L. Clements ${ }^{16}$, A. Conley $^{17}$, C. D. Dowell ${ }^{1,12}$, S. Eales ${ }^{8}$, D. Farrah ${ }^{18}$, A. Franceschini ${ }^{19}$, \\ R. Gavazzi ${ }^{3}$, J. GlenN ${ }^{17}$, M. Griffin ${ }^{8}$, M. Gurwell ${ }^{11}$, R. J. Ivison ${ }^{20,21}$, S. Kim ${ }^{2}$, M. Krips ${ }^{4}$, A. M. J. Mortier ${ }^{16}$, S. J. Oliver ${ }^{18}$, \\ M. J. Page ${ }^{22}$, A. Papageorgiou ${ }^{8}$, C. P. Pearson ${ }^{23,24}$, I. Pérez-Fournon ${ }^{25,26}$, M. Pohlen ${ }^{8}$ J. I. RaWlings $^{22}$, G. Raymond $^{8}$, \\ G. Rodighiero ${ }^{19}$, I. G. Roseboom ${ }^{18}$, M. Rowan-Robinson ${ }^{16}$, K. S. ScotT ${ }^{27}$, N. Seymour ${ }^{22}$, A. J. Smith ${ }^{18}$, M. Symeonidis ${ }^{22}$,

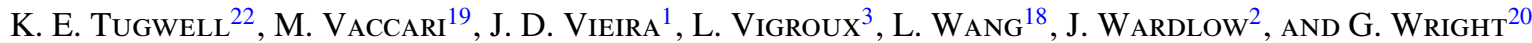 \\ ${ }^{1}$ Astronomy Department, California Institute of Technology, MC 249-17, 1200 East California Boulevard, Pasadena, CA 91125, USA; dr@caltech.edu \\ ${ }^{2}$ Department of Physics \& Astronomy, University of California, Irvine, CA 92697, USA \\ ${ }^{3}$ Institut d'Astrophysique de Paris, CNRS and Université Pierre et Marie Curie, 98 bis Bd Arago, F-75014 Paris, France \\ ${ }^{4}$ Institut de RadioAstronomie Millimétrique, 300 Rue de la Piscine, Domaine Universitaire, F-38406 Saint Martin d'Héres, France \\ ${ }^{5}$ Department of Astronomy, University of Maryland, College Park, MD 20742-2421, USA \\ ${ }^{6}$ Department of Physics and Astronomy, Rutgers, the State University of New Jersey, 136 Frelinghuysen Road, Piscataway, NJ 08854-8019, USA \\ ${ }^{7}$ National Radio Astronomy Observatory, P.O. Box 2, Green Bank, WV 24944, USA \\ ${ }^{8}$ School of Physics and Astronomy, Cardiff University, Queens Buildings, The Parade, Cardiff CF24 3AA, UK \\ ${ }^{9}$ Laboratoire AIM-Paris-Saclay, CEA/DSM/Irfu-CNRS-Université Paris Diderot, CE-Saclay, pt courrier 131, F-91191 Gif-sur-Yvette, France \\ ${ }^{10}$ Institut d'Astrophysique Spatiale, Université Paris-Sud XI, bât 121, F-91405 Orsay Cedex, France \\ ${ }^{11}$ Harvard-Smithsonian Center for Astrophysics, Cambridge, MA 02138, USA \\ 12 Jet Propulsion Laboratory, 4800 Oak Grove Drive, Pasadena, CA 91109, USA \\ ${ }^{13}$ Space Science Building, Cornell University, Ithaca, NY, 14853-6801, USA \\ ${ }^{14}$ Laboratoire d'Astrophysique de Marseille, OAMP, Université Aix-marseille, CNRS, 38 rue Frédéric Joliot-Curie, F-13388 Marseille Cedex 13, France \\ ${ }_{15}^{15}$ Institute of Astronomy, University of Cambridge, Madingley Road, Cambridge CB3 OHA, UK \\ ${ }^{16}$ Astrophysics Group, Imperial College London, Blackett Laboratory, Prince Consort Road, London SW7 2AZ, UK \\ ${ }^{17}$ Department of Astrophysical and Planetary Sciences, CASA 389-UCB, University of Colorado, Boulder, CO 80309, USA \\ ${ }^{18}$ Astronomy Centre, Department of Physics \& Astronomy, University of Sussex, Brighton BN1 9QH, UK \\ ${ }^{19}$ Dipartimento di Astronomia, Università di Padova, Vicolo Osservatorio, 3, I-35122 Padova, Italy \\ ${ }^{20}$ UK Astronomy Technology Centre, Royal Observatory, Blackford Hill, Edinburgh EH9 3HJ, UK \\ ${ }^{21}$ Institute for Astronomy, University of Edinburgh, Royal Observatory, Blackford Hill, Edinburgh EH9 3HJ, UK \\ ${ }^{22}$ Mullard Space Science Laboratory, University College London, Holmbury St. Mary, Dorking, Surrey RH5 6NT, UK \\ ${ }^{23}$ Space Science \& Technology Department, Rutherford Appleton Laboratory, Chilton, Didcot, Oxfordshire OX11 0QX, UK \\ ${ }^{24}$ Institute for Space Imaging Science, University of Lethbridge, Lethbridge, Alberta, T1K 3M4, Canada \\ ${ }^{25}$ Instituto de Astrofísica de Canarias, E-38200 La Laguna, Tenerife, Spain \\ ${ }^{26}$ Departamento de Astrofísica, Universidad de La Laguna, E-38205 La Laguna, Tenerife, Spain \\ ${ }^{27}$ Department of Physics and Astronomy, University of Pennsylvania, Philadelphia, PA 19104, USA \\ Received 2011 February 18; accepted 2011 April 14; published 2011 May 3
}

\begin{abstract}
We report the detection of $\mathrm{CO}(J=5 \rightarrow 4), \mathrm{CO}(J=3 \rightarrow 2)$, and $\mathrm{CO}(J=1 \rightarrow 0)$ emission in the strongly lensed, Herschel/SPIRE-selected submillimeter galaxy (SMG) HERMES J105751.1+573027 at $z=$ $2.9574 \pm 0.0001$, using the Plateau de Bure Interferometer, the Combined Array for Research in Millimeterwave Astronomy, and the Green Bank Telescope. The observations spatially resolve the molecular gas into four lensed images with a maximum separation of $\sim 9^{\prime \prime}$ and reveal the internal gas dynamics in this system. We derive lensing-corrected $\mathrm{CO}$ line luminosities of $L_{\mathrm{CO}(1-0)}^{\prime}=(4.17 \pm 0.41), L_{\mathrm{CO}(3-2)}^{\prime}=(3.96 \pm 0.20)$, and $L_{\mathrm{CO}(5-4)}^{\prime}=(3.45 \pm 0.20) \times 10^{10}\left(\mu_{\mathrm{L}} / 10.9\right)^{-1} \mathrm{~K} \mathrm{~km} \mathrm{~s}^{-1} \mathrm{pc}^{2}$, corresponding to luminosity ratios of $r_{31}=$ $0.95 \pm 0.10, r_{53}=0.87 \pm 0.06$, and $r_{51}=0.83 \pm 0.09$. This suggests a total molecular gas mass of $M_{\text {gas }}=$ $3.3 \times 10^{10}\left(\alpha_{\mathrm{CO}} / 0.8\right)\left(\mu_{\mathrm{L}} / 10.9\right)^{-1} M_{\odot}$. The gas mass, gas mass fraction, gas depletion timescale, star formation efficiency, and specific star formation rate are typical for an SMG. The velocity structure of the gas reservoir suggests that the brightest two lensed images are dynamically resolved projections of the same dust-obscured region in the galaxy that are kinematically offset from the unresolved fainter images. The resolved kinematics appear consistent with the complex velocity structure observed in major, "wet" (i.e., gas-rich) mergers. Major mergers are commonly observed in SMGs and are likely to be responsible for fueling their intense starbursts at high gas consumption rates. This study demonstrates the level of detail to which galaxies in the early universe can be studied by utilizing the increase in effective spatial resolution and sensitivity provided by gravitational lensing.
\end{abstract}

Key words: cosmology: observations - galaxies: active - galaxies: formation - galaxies: high-redshift - galaxies: starburst - radio lines: galaxies

Online-only material: color figures

\footnotetext{
${ }^{28}$ Hubble Fellow
}

\section{INTRODUCTION}

Great progress has been made over the past decade in understanding the role of submillimeter-selected galaxies (SMGs; 
see reviews by Blain et al. 2002; Lagache et al. 2005; Smail 2006) in the early evolution of massive galaxies. Studies of the stellar populations of massive galaxies suggest that they form the bulk of their stars at early epochs $(z>2)$, with the more massive galaxies building up their stellar component at earlier epochs and on shorter timescales (see review by Renzini 2006). These findings suggest the existence of a population of massive, gas-rich starburst galaxies at early cosmic times-properties which are consistent with those of SMGs. SMGs are typically intense $\left(\gtrsim 500 M_{\odot} \mathrm{yr}^{-1}\right)$, optically obscured, relatively shortlived $(<100 \mathrm{Myr})$ starbursts at high redshift, implying rapid gas consumption timescales at high star formation efficiencies (e.g., Smail et al. 1997; Hughes et al. 1998; Greve et al. 2005). Most SMGs have sizes of a few kpc and are dynamically complex, exhibiting the properties of gas-rich, major mergers (e.g., Tacconi et al. 2008).

The physical properties of the star-forming material in these extreme galaxies are best studied using rotational emission lines of molecular gas tracers, most commonly CO. Molecular gas is the fuel for star formation and has been detected in > 30 SMGs to date. These typically have large gas reservoirs of $>10^{10} M_{\odot}$ (see review by Solomon \& Vanden Bout 2005). The difficulty of such studies is reflected in the moderate number of detections and limited level of detail of most experiments. A promising way to overcome these limitations is to study strongly gravitationally lensed SMGs, as lensing provides a boost in sensitivity and spatial resolution. However, strongly lensed SMGs are very rare, making them difficult to find in typical submillimeter continuum surveys.

With the advent of large area $\left(\gg 1 \mathrm{deg}^{2}\right)$ submillimeter surveys that are currently being undertaken with Herschel such as the Multi-tiered Extragalactic Survey (HerMES; S. Oliver et al. 2011, in preparation), ${ }^{29}$ efficient identification of lensed SMGs is now becoming possible. Here, we study the molecular gas properties of the brightest source identified in these studies.

In this Letter, we report the detection of spatially resolved $\mathrm{CO}(J=5 \rightarrow 4)$ and $\mathrm{CO}(J=3 \rightarrow 2)$ emission and of $\mathrm{CO}(J=1 \rightarrow 0)$ emission toward the strongly lensed SMG HERMES J105751.1+573027 (hereafter HLSW-01; $z=2.957$ ), using the Plateau de Bure Interferometer (PdBI), the Combined Array for Research in Millimeter-wave Astronomy (CARMA), and the $100 \mathrm{~m}$ Green Bank Telescope (GBT). HLSW-01 was discovered in the HerMES Herschel/SPIRE Science Demonstration Phase (SDP) data (Oliver et al. 2010) and is the brightest $z>2$ SMG currently known. Its spectral energy distribution peaks at an (apparent) $250 \mu \mathrm{m}$ flux of $425 \pm 10 \mathrm{mJy}$ (Conley et al. 2011, hereafter C11). It is lensed by a galaxy group at $z_{\mathrm{G}}=$ $0.60 \pm 0.04$, magnifying the SMG by a factor of $\mu_{\mathrm{L}}=10.9 \pm 0.7$ in flux density (Gavazzi et al. 2011, hereafter G11). In accompanying work, we provide additional details on the identification, molecular line excitation (based on this work and $\mathrm{CO} J=7 \rightarrow 6$ to $J=10 \rightarrow 9$ observations with $\mathrm{CSO} / \mathrm{Z}$-spec; Scott et al. 2011, hereafter S11), spectral energy distribution (C11), and lensing properties (G11) of HLSW-01. We use a concordance, flat $\Lambda$ CDM cosmology throughout, with $H_{0}=71 \mathrm{~km} \mathrm{~s}^{-1} \mathrm{Mpc}^{-1}$, $\Omega_{\mathrm{M}}=0.27$, and $\Omega_{\Lambda}=0.73$ (Spergel et al. 2003, 2007).

\section{OBSERVATIONS}

\subsection{Plateau de Bure Interferometer}

We observed the $\mathrm{CO}(J=5 \rightarrow 4)$ transition line $\left(v_{\text {rest }}=\right.$ $576.2679305 \mathrm{GHz}$, redshifted to $145.633 \mathrm{GHz}$, or $2.06 \mathrm{~mm}$ )

\footnotetext{
29 http://hermes.sussex.ac.uk/
}

toward HLSW-01 $(z=2.957)$, using the PdBI. We used the WideX correlator with a total bandwidth of $3.6 \mathrm{GHz}$ $\left(\sim 7400 \mathrm{~km} \mathrm{~s}^{-1}\right.$, at $1.95 \mathrm{MHz}$ resolution) to cover the $\mathrm{CO}(J=$ $5 \rightarrow 4$ ) line and the underlying $2 \mathrm{~mm}$ (rest-frame $520 \mu \mathrm{m}$ ) continuum emission. Observations were carried out under good $2 \mathrm{~mm}$ weather conditions for two tracks in 5D configuration on 2010 June 14 and 17 . This resulted in $1.0 \mathrm{hr}$ of six antennaequivalent on-source time after discarding unusable visibility data. The nearby (10.6 distant) source B0954+658 (J0958+655; $0.98 \mathrm{Jy}$ ) was observed every 20 minutes for pointing, amplitude, and phase calibration. MWC 349 (1.50 Jy) and 3C273 (10.5 Jy) were observed for flux and bandpass calibration, yielding $\sim 10 \%$ calibration accuracy.

The IRAM GILDAS package was used for data reduction and analysis. All data were mapped using the CLEAN algorithm with "natural" weighting, resulting in a synthesized beam of 4 ". $6 \times 2$ ". 8 . The final $\mathrm{rms}$ is $1.2 \mathrm{mJy}^{\text {beam }}{ }^{-1}$ over $250 \mathrm{MHz}$ (corresponding to $515 \mathrm{~km} \mathrm{~s}^{-1}$ ), $3.4 \mathrm{mJy}$ beam $^{-1}$ over $31.25 \mathrm{MHz}$ $\left(64 \mathrm{~km} \mathrm{~s}^{-1}\right)$, and $6.0 \mathrm{mJy}^{\text {beam }}{ }^{-1}$ over $10 \mathrm{MHz}\left(21 \mathrm{~km} \mathrm{~s}^{-1}\right)$.

\subsection{CARMA}

We observed the $\operatorname{CO}(J=3 \rightarrow 2)$ transition line $\left(v_{\text {rest }}=\right.$ $345.7959899 \mathrm{GHz}$, redshifted to $87.388 \mathrm{GHz}$, or $3.43 \mathrm{~mm}$ ), using CARMA. A total bandwidth of $3.7 \mathrm{GHz}\left(\sim 12,700 \mathrm{~km} \mathrm{~s}^{-1}\right.$, at $5.208 \mathrm{MHz}$ resolution) was used to cover the $\mathrm{CO}(J=$ $3 \rightarrow 2$ ) line and the underlying $3.4 \mathrm{~mm}$ (rest-frame $870 \mu \mathrm{m}$ ) continuum emission. Observations were carried out under good $3 \mathrm{~mm}$ weather conditions (typically at moderate elevations after transit) for six tracks in D configuration between 2010 September 01 and 17. This resulted in $6.6 \mathrm{hr}$ of 15 antennaequivalent on-source time after discarding unusable visibility data. The nearby source J0958+655 $(0.86 \mathrm{Jy})$ was observed every 15 minutes for pointing, amplitude, and phase calibration. Fluxes were bootstrapped relative to Mars (based on a brightness temperature model of the planet). Several nearby calibrators (3C 84, 3C 273, 3C 345, J0927+390, J2015+372) were observed for bandpass calibration, yielding $\sim 15 \%$ calibration accuracy.

The MIRIAD package was used for data reduction and analysis. All data were mapped using the CLEAN algorithm with "natural" weighting, resulting in a synthesized beam of 7 ".7 $\times 5^{\prime \prime}$.0. The final $\mathrm{rms}$ is $0.48 \mathrm{mJy}^{\text {beam }}{ }^{-1}$ over $226 \mathrm{MHz}$ (corresponding to $777 \mathrm{~km} \mathrm{~s}^{-1}$ ), $1.6 \mathrm{mJy}_{\text {beam }}^{-1}$ over $20.8 \mathrm{MHz}$ $\left(71 \mathrm{~km} \mathrm{~s}^{-1}\right)$, and $2.3 \mathrm{mJy}^{\text {beam }}{ }^{-1}$ over $10.4 \mathrm{MHz}\left(36 \mathrm{~km} \mathrm{~s}^{-1}\right)$.

\section{3. $G B T$}

We observed the $\operatorname{CO}(J=1 \rightarrow 0)$ transition line $\left(v_{\text {rest }}=\right.$ $115.2712018 \mathrm{GHz}$, redshifted to $29.131 \mathrm{GHz}$, or $10.29 \mathrm{~mm}$ ), using the Zpectrometer analog lag cross-correlation spectrometer (Harris et al. 2007) attached to the GBT Ka band receiver. This yields an instantaneous bandwidth of $10.5 \mathrm{GHz}$ $\left(\sim 108,000 \mathrm{~km} \mathrm{~s}^{-1}\right)$ at a beam size of $27^{\prime \prime}-19^{\prime \prime}(25.6-36.1 \mathrm{GHz})$. Observations were carried out under good $1 \mathrm{~cm}$ weather conditions on 2010 October 2. This resulted in $1.5 \mathrm{hr}$ on-source time after discarding unusable data. Subreflector beam switching was used every $10 \mathrm{~s}$ to observe the source alternately with the receiver's two beams, with the off-source beam monitoring the sky background in parallel. A nearby second, fainter source was observed with the same pattern, switching between targets with 8 minute cycles. Residual structure from optical beam imbalance in the "source-sky" difference spectra of each target was then removed by differencing both sources. A spectral ripple with well-defined period generated in the receiver 


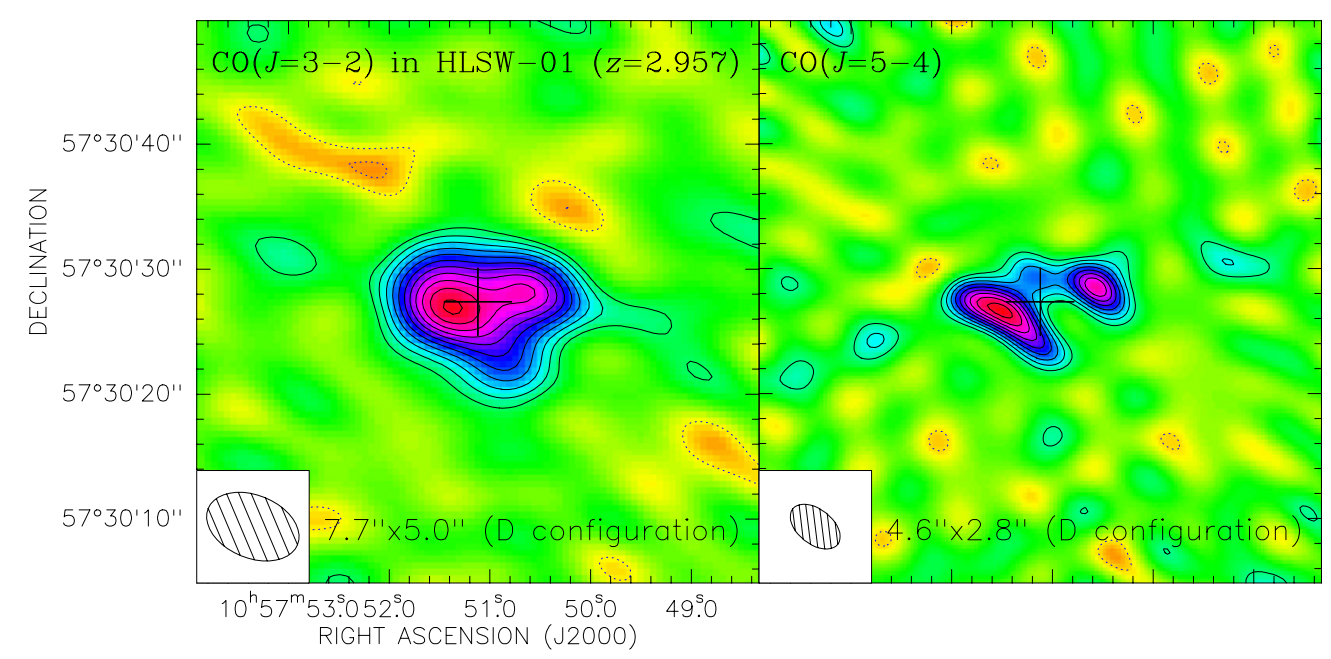

Figure 1. Velocity-integrated CARMA and PdBI maps of $\mathrm{CO}(J=3 \rightarrow 2)$ and $\mathrm{CO}(J=5 \rightarrow 4)$ line emission over 777 and $515 \mathrm{~km} \mathrm{~s}{ }^{-1}$ toward HLSW- 01 . At 7 ". $^{\prime} \times 5^{\prime \prime} .0$ and 4". $6 \times 2$ '. 8 resolution (as indicated in the bottom left), the emission is clearly resolved. The crosses indicate the center of the $250 \mu \mathrm{m}$ emission (C11). Contours are shown in steps of $1 \sigma=0.48$ and $1.2 \mathrm{mJy}^{-1}$ beam ${ }^{-1}$, starting at $\pm 2 \sigma$.

(A color version of this figure is available in the online journal.)

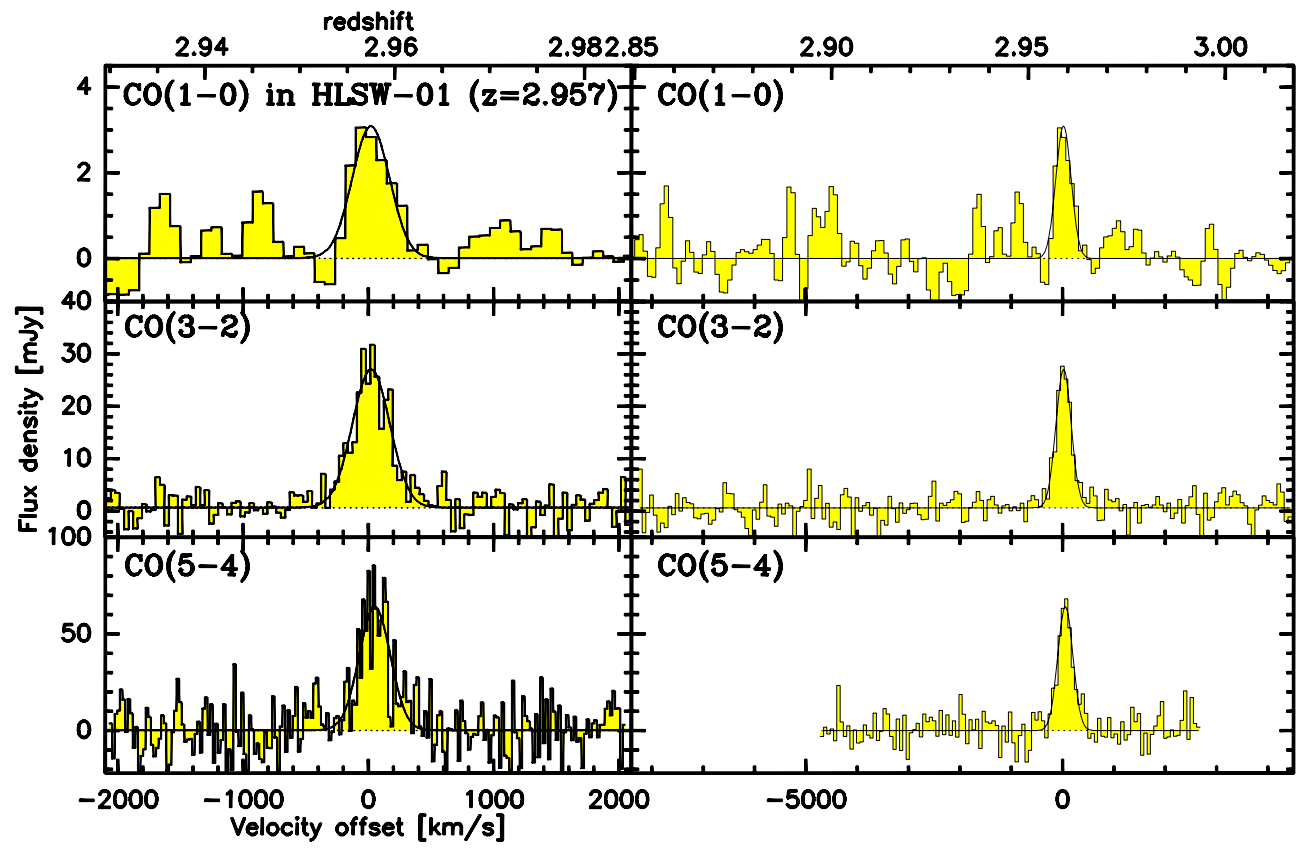

Figure 2. Left: $\mathrm{CO}(J=1 \rightarrow 0), \mathrm{CO}(J=3 \rightarrow 2)$, and $\mathrm{CO}(J=5 \rightarrow 4)$ spectra of HLSW-01 at 82/36/21 km s${ }^{-1}(8.0 / 10.416 / 10.0 \mathrm{MHz})$ resolution (histograms), along with Gaussian fits to the line and continuum emission (black curves). The velocity scale is relative to $z=2.957$. The $\mathrm{CO}(J=1 \rightarrow 0)$ channels are not statistically independent on scales below $20 \mathrm{MHz}\left(2.5\right.$ channels). Right: same, re-binned to similar velocity resolution $\left(82 / 71 / 64 \mathrm{~km} \mathrm{~s}{ }^{-1}\right.$ or $8.0 / 20.832 / 31.25 \mathrm{MHz}$; CO $J=$ $1 \rightarrow 0$ unchanged) and showing a larger velocity range.

(A color version of this figure is available in the online journal.)

was removed with narrowband Fourier filtering. This strategy yields a flat baseline (offset from zero flux by to the difference of the two faint source continua) without standard polynomial baseline removal. The nearby quasar $1143+4931$ was observed every $\sim 1$ hr to monitor telescope pointing and gain stability. Passband gains and absolute fluxes were determined from spectra of 3C286 (2.04 Jy at $32 \mathrm{GHz}$; Ott et al. 1994), yielding $15 \%$ calibration accuracy. ${ }^{30}$

The lag data were processed using the Zpectrometer's data reduction pipeline and standard recipes, yielding a frequencycalibrated spectrum at $8 \mathrm{MHz}$ resolution (see Harris et al.

\footnotetext{
30 The 2010 Astronomical Almanac suggests a slightly lower value of $1.95 \mathrm{Jy}$
} at $29 \mathrm{GHz}$ for $3 \mathrm{C} 286$.
2010 for details). The instrumental spectral response is nearly a sinc function with an FWHM of $20 \mathrm{MHz}$, i.e., individual $8 \mathrm{MHz}$ spectral channels are not statistically independent. However, the linewidth correction for the instrumental response for spectral lines with intrinsic Gaussian FWHM of $>30 \mathrm{MHz}$ $\left(\sim 300 \mathrm{~km} \mathrm{~s}^{-1}\right)$ is minor.

\section{RESULTS}

\subsection{Integrated Emission Line and Continuum Properties}

We have detected spatially resolved $\mathrm{CO}(J=3 \rightarrow 2)$ and $\mathrm{CO}(J=5 \rightarrow 4)$ (Figure 1$)$ and unresolved $\mathrm{CO}(J=1 \rightarrow 0)$ line emission toward HLSW-01 at high significance. From Gaussian fitting to the (spatially integrated) line profiles (Figure 2), we 


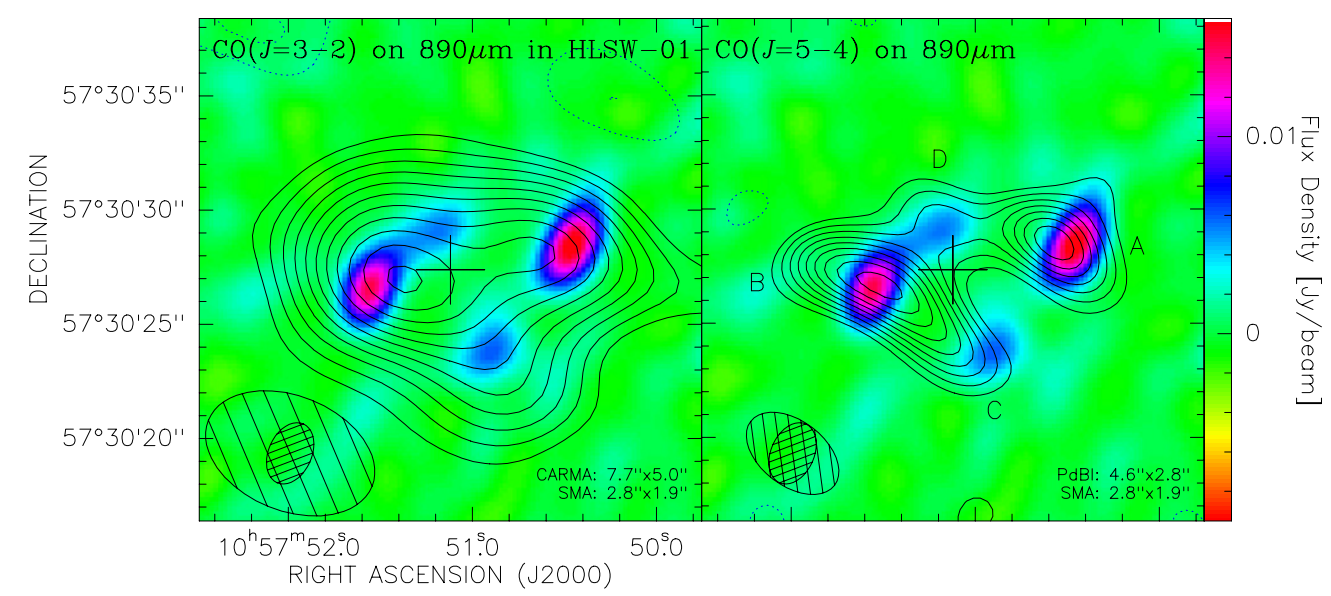

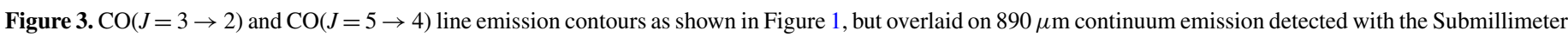

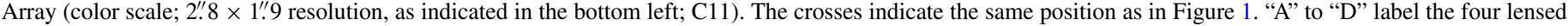
images of the source ordered by brightness of the $890 \mu \mathrm{m}$ continuum emission. CO emission is detected toward all four images.

(A color version of this figure is available in the online journal.)

obtain $\mathrm{CO}(J=1 \rightarrow 0), \mathrm{CO}(J=3 \rightarrow 2)$, and $\mathrm{CO}(J=5 \rightarrow 4)$ line peak strengths of $3.09 \pm 0.37,26.4 \pm 1.5$, and $64.0 \pm$ $4.4 \mathrm{mJy}$ at a median FWHM of $348 \pm 18 \mathrm{~km} \mathrm{~s}^{-1}(350 \pm 55$, $350 \pm 25$, and $346 \pm 29 \mathrm{~km} \mathrm{~s}^{-1}$ for the $\mathrm{CO} \mathrm{J}=1 \rightarrow 0,3 \rightarrow 2$, and $5 \rightarrow 4$ lines). We marginally detect $3.43 \mathrm{~mm}$ continuum emission under the $\mathrm{CO}(J=3 \rightarrow 2)$ line at $0.61 \pm 0.19 \mathrm{mJy}$ strength, and do not detect $2.06 \mathrm{~mm}$ continuum emission down to a $2 \sigma$ limit of $1.4 \mathrm{mJy}$. By combining all lines, we find a median redshift of $z=2.9574 \pm 0.0001$. The line parameters correspond to velocity-integrated emission line strengths of $1.14 \pm 0.11$, $9.74 \pm 0.49$, and $23.6 \pm 1.4 \mathrm{Jy} \mathrm{km} \mathrm{s}^{-1}$, and line luminosities of $L_{\mathrm{CO}(1-0)}^{\prime}=(4.17 \pm 0.41), L_{\mathrm{CO}(3-2)}^{\prime}=(3.96 \pm 0.20)$, and $L_{\mathrm{CO}(5-4)}^{\prime}=(3.45 \pm 0.20) \times 10^{10}\left(\mu_{\mathrm{L}} / 10.9\right)^{-1} \mathrm{~K} \mathrm{~km} \mathrm{~s}^{-1} \mathrm{pc}^{2}$, respectively. This corresponds to line brightness temperature ratios of $r_{31}=0.95 \pm 0.10, r_{53}=0.87 \pm 0.06$, and $r_{51}=0.83 \pm$ 0.09 . This suggests that at least the $\operatorname{CO}(J=5 \rightarrow 4)$ line is not thermalized (i.e., not in radiative equilibrium with the lower$J$ lines; $r<1$; see also S11). We do not find evidence for a substantial low-excitation gas component in HLSW-01 (i.e., gas with $r_{31} \ll 1$ ), contrary to what is found in some other $z>2$ SMGs (e.g., Carilli et al. 2010; Riechers et al. 2010; Harris et al. 2010; Ivison et al. 2010a). We estimate the total molecular gas mass assuming a conversion factor of $\alpha_{\mathrm{CO}}=0.8 M_{\odot}\left(\mathrm{K} \mathrm{km} \mathrm{s}^{-1}\right.$ $\left.\mathrm{pc}^{2}\right)^{-1}$ from $L_{\mathrm{CO}(1-0)}^{\prime}$ to $M_{\text {gas }}$, as appropriate for ultra-luminous infrared galaxies (ULIRGs; Downes \& Solomon 1998) and commonly used for SMGs. This yields $M_{\mathrm{gas}}=3.3 \times 10^{10}\left(\alpha_{\mathrm{CO}} /\right.$ 0.8) $\left(\mu_{\mathrm{L}} / 10.9\right)^{-1} M_{\odot}$.

\subsection{Limits on Dense Gas Tracers}

Several lines of dense molecular gas tracers fall within the spectral range covered by the $\mathrm{CO}(J=3 \rightarrow 2)$ and $\mathrm{CO}(J=5 \rightarrow 4)$ observations. We obtain $3 \sigma$ upper limits of $<1.6 \mathrm{Jy} \mathrm{km} \mathrm{s}^{-1}$ on the integrated $\operatorname{HCN}(J=4 \rightarrow 3), \operatorname{CS}(J=7 \rightarrow 6), \operatorname{SiO}(J=$ $8 \rightarrow 7)$, and $\mathrm{HC}_{3} \mathrm{~N}(J=39 \rightarrow 38)$ emission line strengths, and $<3.2 \mathrm{Jy} \mathrm{km} \mathrm{s}^{-1}$ for $\mathrm{HC}_{3} \mathrm{~N}(J=63 \rightarrow 62)$ and $\mathrm{HC}_{3} \mathrm{~N}(J=$ $64 \rightarrow 63$ ) (averaged over $348 \mathrm{~km} \mathrm{~s}^{-1}$ ). This corresponds to line luminosity limits of $<0.61,<0.66,<0.64,<0.61,<0.48$, and $<0.46 \times 10^{10}\left(\mu_{\mathrm{L}} / 10.9\right)^{-1} \mathrm{~K} \mathrm{~km} \mathrm{~s}^{-1} \mathrm{pc}^{2}$ for the HCN, CS, SiO, $\mathrm{HC}_{3} \mathrm{~N}(J=39 \rightarrow 38), \mathrm{HC}_{3} \mathrm{~N}(J=63 \rightarrow 62)$, and $\mathrm{HC}_{3} \mathrm{~N}(J=$ $64 \rightarrow 63)$ lines, respectively. This also corresponds to line brightness temperature ratio limits of $<0.15,<0.16,<0.15$, $<0.15,<0.11$, and $<0.11$ relative to $\operatorname{CO}(J=1 \rightarrow 0)$. Nearby starburst galaxies and ULIRGs like NGC 253, NGC 6240, and Arp 220 typically have $\mathrm{HCN} J=4 \rightarrow 3 / J=1 \rightarrow 0$ line ratios of 0.45-0.8 (Knudsen et al. 2007; Greve et al. 2009). If this ratio is similar in HLSW-01, it suggests an $\mathrm{HCN} / \mathrm{CO}$ ratio of $<0.18-0.33$ in the respective $J=1 \rightarrow 0$ transitions, consistent with what is found in nearby ULIRGs and other high- $z$ gas-rich galaxies (median ratios of 0.17; Gao \& Solomon 2004a, 2004b; Gao et al. 2007; Riechers et al. 2007).

\subsection{Morphology of the Molecular Gas Reservoir}

We spatially resolve the (lensed) molecular gas reservoir in HLSW-01 in the $\mathrm{CO}(J=3 \rightarrow 2)$ and $\mathrm{CO}(J=5 \rightarrow 4)$ emission lines. The characteristic effective resolution provided by the lensing effect is $\sim 2$ ". 2 (i.e., regions of $\sim 8.5 \mathrm{kpc}$ radius) in $\mathrm{CO}(J=5 \rightarrow 4)$, but substantially higher in the brighter images and close to critical lines (G11). Overlays of the CO emission with $890 \mu \mathrm{m}$ (rest-frame $225 \mu \mathrm{m}$ ) continuum emission are shown in Figure 3. The rest-frame far-infrared (FIR) continuum emission dominantly traces the regions of most active star formation in this system (star formation rate: $2460 \pm 160$ $\left.\left(\mu_{\mathrm{L}} / 10.9\right)^{-1} M_{\odot} \mathrm{yr}^{-1} ; \mathrm{C} 11\right)$. The $\mathrm{CO}(J=5 \rightarrow 4)$ emission shows discernible peaks at the positions of lens images $\mathrm{A}, \mathrm{B}$, and $\mathrm{D}$, with an extension of the emission associated with image $\mathrm{B}$ toward image $\mathrm{C}$. Accounting for beam convolution, there is no evidence for differences in the spatial distribution and/or line shapes of the $\operatorname{CO}(J=3 \rightarrow 2)$ and $\operatorname{CO}(J=5 \rightarrow 4)$ emission, indicating that both lines trace the same material. The small offsets between the $\mathrm{CO}$ and FIR peaks (typically $<10 \%$ of the relative beam sizes) are a combination of beam convolution (given the different beam sizes and orientations) and velocityaveraged dynamical structure in the integrated molecular line maps. The molecular gas reservoir may extend beyond the FIR continuum peaks, but maps at higher spatial resolution and sensitivity are required to investigate this in more detail. The overall striking match between the gas and (FIR) dust emission suggests that, indeed, the bulk of the FIR light is associated with the gas-rich, star-forming regions.

An overlay of the $\mathrm{CO}(J=5 \rightarrow 4)$ emission with $2.2 \mu \mathrm{m}$ (rest-frame $560 \mathrm{~nm}$; smoothed with a 0.1 Gaussian kernel) continuum emission is shown in Figure 4. The $\mathrm{CO}$ emission is clearly associated with the faint optical lensed images of the SMG, which appear to consist of multiple clumps. However, 


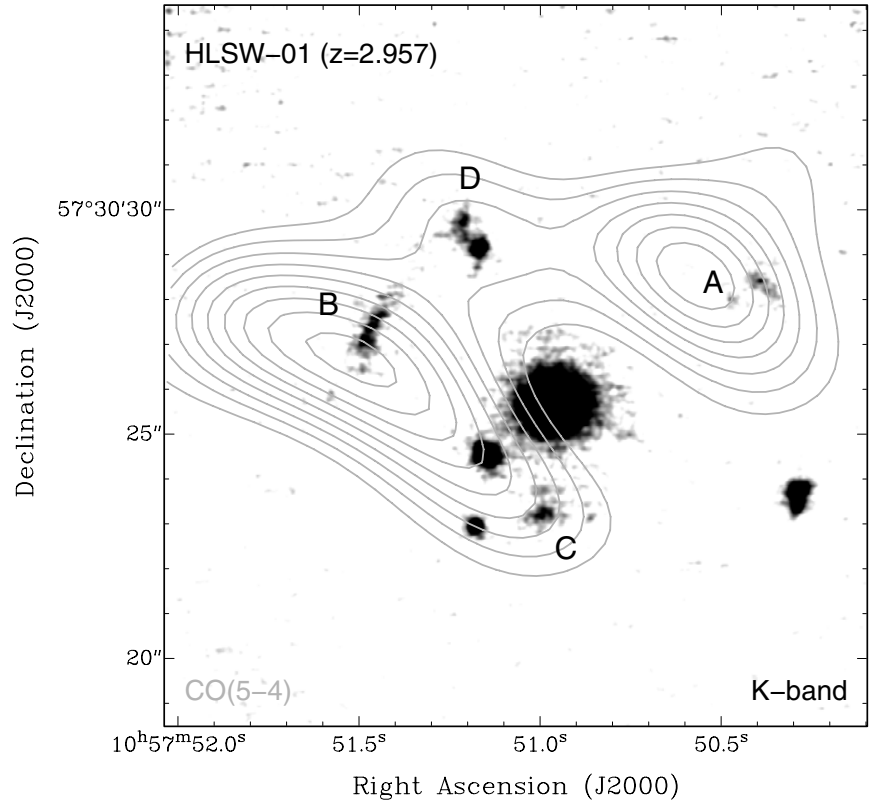

Figure 4. $\mathrm{CO}(J=5 \rightarrow 4)$ line emission contours as shown in Figure 1, but overlaid on $2.2 \mu \mathrm{m}$ continuum emission (gray scale; smoothed with a $0^{\prime \prime} 1$ Gaussian kernel; C11). "A" to "D" label the four lensed images as in Figure 3. The CO emission is associated with the four rest-frame optical lensed images, but the peaks of the emission are optically faint.

the brightest $\mathrm{CO}$ peaks are slightly offset from the peaks of the rest-frame optical emission. This offset translates to $\sim 2.4 \mathrm{kpc}$ in the source plane after correcting for gravitational magnification (G11). This may indicate that the most gas-rich regions are optically obscured and/or the presence of multiple galaxy components, as commonly observed in SMGs. Also, the FIRbrightest lens image $\mathrm{A}$ is the optically faintest image of the galaxy, which lends further support to this picture.

\subsection{Dynamical Structure of the Gas Reservoir}

A first moment map of the $\mathrm{CO}(J=5 \rightarrow 4)$ emission is shown in Figure 5 (clipped below $2 \sigma$ in each velocity channel, smoothed before detection). There is a clear velocity gradient between images $\mathrm{A} / \mathrm{B}$ and $\mathrm{C} / \mathrm{D}$. The peak of image $\mathrm{D}$ is blueshifted by up to $\sim 380 \mathrm{~km} \mathrm{~s}^{-1}$ (relative to zero velocity in Figure 2). The peak of image $\mathrm{C}$ is blended with image $\mathrm{B}$, but is consistent with being redshifted by up to $\sim 150 \mathrm{~km} \mathrm{~s}^{-1}$. Images $\mathrm{A}$ and $\mathrm{B}$ peak on the line center. Image A shows a smooth north-south velocity gradient from red to blue, and image B shows a gradient in the opposite direction. This gradient is also seen in $\mathrm{CO}(J=3 \rightarrow 2)$ emission, indicating that these are likely dynamically resolved lensed images of the same region in the galaxy. Images $\mathrm{C}$ and $\mathrm{D}$ are consistent with being unresolved, (in their peaks) kinematically distinct regions in the same galaxy or a companion system. The complexity of the velocity structure is unlikely to be due to distortion of the velocity field by gravitational lensing alone, but would be consistent with the dynamical structure of a major, "wet" (i.e., gas-rich) merger in progress (e.g., Tacconi et al. 2008; Engel et al. 2010).

Based on the velocity-integrated $\mathrm{CO}(J=5 \rightarrow 4)$ map shown in Figure 1, G11 find a half-flux radius of $1.1 \pm 0.5 \mathrm{kpc}$ for the CO reservoir in HLSW-01, after correcting for gravitational magnification. However, the structure seen in the $\mathrm{CO}$ velocity map indicates that this size estimate may be a lower limit. Thus, different parts of the $\mathrm{CO}$ emission may be differentially magnified. However, the lens model by G11 suggests that

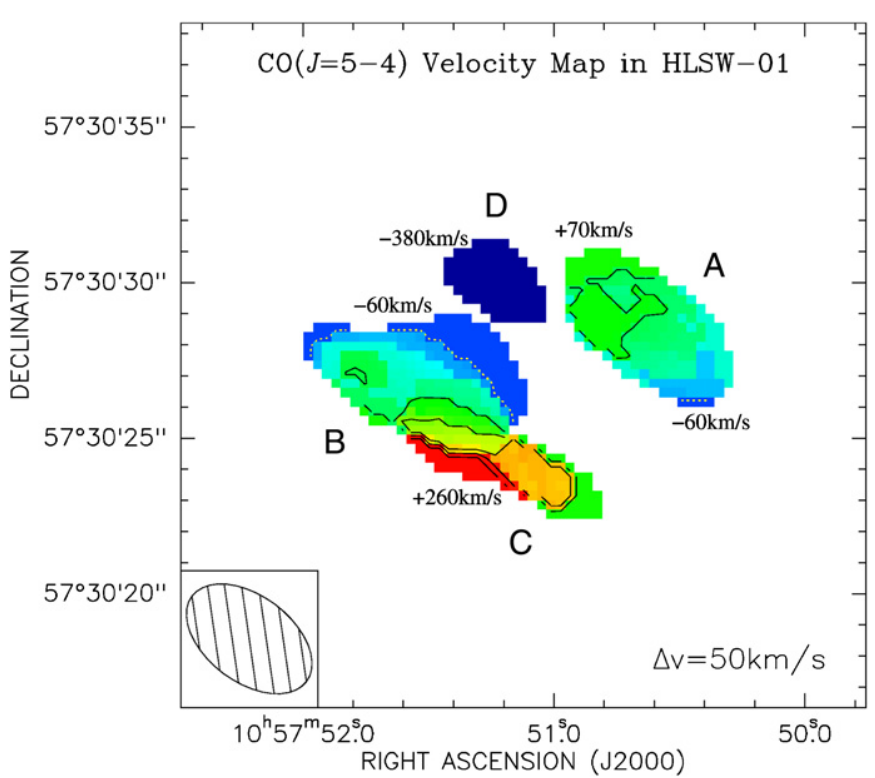

Figure 5. $\mathrm{CO}(J=5 \rightarrow 4)$ first moment map. The colors indicate the velocity gradient. Contours are shown in steps of $50 \mathrm{~km} \mathrm{~s}^{-1}$, with dotted (solid) contours showing blueshifted (redshifted) emission relative to zero velocity in Figure 2. "A" to "D" label the four lensed images as in Figure 3. Images A and B are blueshifted relative to image $\mathrm{C}$ and redshifted relative to image $\mathrm{D}$. Image $\mathrm{A}$ shows a north-south velocity gradient (red to blue), and image B shows a gradient in the opposite direction. No velocity gradients are resolved toward images $\mathrm{C}$ and $\mathrm{D}$.

differential lensing effects are only moderate $(\lesssim 25 \%)$, even out to a radius of $10 \mathrm{kpc}$ (their Figure 3), and, thus, have a relatively minor effect on the integrated gas properties.

\subsection{Physical Properties of the Gas, Dust, and Stellar Components}

The minimum time for which the starburst in HLSW-01 can be maintained at its current rate is given by the gas depletion timescale ${ }^{31} \tau_{\text {dep }}^{0.8}=M_{\text {gas }} / \mathrm{SFR} \sim 14 \pm 2 \mathrm{Myr}$, which is short but within the range of values found for "typical" SMGs (e.g., Greve et al. 2005).

The ratio between $L_{\mathrm{IR}}(\propto \mathrm{SFR})$ and $L_{\mathrm{CO}}^{\prime}\left(\propto M_{\mathrm{gas}}\right)$ can be used as a measure of the star formation efficiency. Using $L_{\mathrm{IR}}=$ $(1.43 \pm 0.09) \times 10^{13}\left(\mu_{\mathrm{L}} / 10.9\right)^{-1} L_{\odot}(\mathrm{C} 11)$, we find a ratio of $\sim 340 \pm 40$, comparable to what is found in "typical" SMGs (e.g., Tacconi et al. 2006; Riechers et al. 2010).

HLSW-01 has a dust mass in the range of $M_{\text {dust }}=$ $(1.0-5.2) \times 10^{8}\left(\mu_{\mathrm{L}} / 10.9\right)^{-1} M_{\odot}(\mathrm{C} 11 ; \mathrm{S} 11)$. This corresponds to a gas-to-dust mass ratio of $f_{g d}^{0.8}=M_{\mathrm{gas}} / M_{\mathrm{dust}}=60-330$ and is consistent with the range of values found in other SMGs (e.g., Michalowski et al. 2010).

HLSW-01 has a stellar mass of $M_{\star}=(6.3 \pm$ $3.4) \times 10^{10}\left(\mu_{\mathrm{L}} / 10.9\right)^{-1} M_{\odot}(\mathrm{C} 11)$, yielding a gas mass fraction of $f_{\text {gas }}^{0.8}=M_{\text {gas }} / M_{\star}=0.53 \pm 0.29$, and a baryonic gas mass fraction of $f_{\text {bary }}^{\mathrm{g}, 0.8}=M_{\mathrm{gas}} /\left(M_{\mathrm{gas}}+M_{\star}\right)=0.35 \pm 0.13$. This also yields a specific star formation rate (SSFR, i.e., SFR $/ M_{\star}$ ) of $39 \pm 21 \mathrm{Gyr}^{-1}$, and a stellar mass doubling timescale $\left(M_{\star} / \mathrm{SFR}\right)$ of $26 \pm 14$ Myr. These values are consistent with what is found for other SMGs as well (e.g., Daddi et al. 2009a; Tacconi et al. 2006, 2008).

\footnotetext{
31 An index " 0.8 " indicates a linear scaling with $\alpha_{\mathrm{CO}}$.
} 


\section{DISCUSSION AND CONCLUSIONS}

We have detected spatially resolved, highly excited $\mathrm{CO}(J=$ $3 \rightarrow 2)$ and $\mathrm{CO}(J=5 \rightarrow 4)$ emission and $\mathrm{CO}(J=1 \rightarrow 0)$ emission toward HLSW-01, the $250 \mu \mathrm{m}$ brightest SMG found in the HerMES Herschel/SPIRE SDP data. Based on these observations, we have refined the redshift of HLSW-01 to $z=2.9574 \pm$ 0.0001 . Once corrected for lensing magnification, HLSW-01 has a relatively modest molecular gas mass for an SMG. The $M_{\text {gas }}$ in HLSW-01 is about twice that found for the strongly lensed $z=2.3$ SMG J2135-0102 $\left(M_{\text {gas }}=1.4 \times 10^{10}\left(\alpha_{\mathrm{CO}} / 0.8\right)\right.$ $\left(\mu_{\mathrm{L}} / 32.5\right)^{-1} M_{\odot} ; \mathrm{J} 2135-0102$ has $\sim 0.87$ times the apparent $250 \mu \mathrm{m}$ flux of HLSW-01; Swinbank et al. 2010; Danielson et al. 2011; Ivison et al. 2010b). However, HLSW-01 has a higher CO excitation in its low- to mid- $J$ lines $\left(r_{31}=0.68 \pm 0.03, r_{53}=\right.$ $0.51 \pm 0.02$, and $r_{51}=0.35 \pm 0.02$ in J2135-0102; Danielson et al. 2011). This difference becomes even more dramatic in the high- $J$ CO lines (S11). HLSW-01 also has a higher characteristic dust temperature of $88 \pm 3 \mathrm{~K}$ (J2135-0102: 30-60 K; Swinbank et al. 2010). The higher $\mathrm{CO}$ excitation and dust temperature in HLSW-01 relative to J2135-0102 and other "typical" $z>2$ SMGs may be due to a higher relative importance of heating by an active galactic nucleus (AGN), but more detailed studies are required to constrain the presence of an AGN in HLSW-01. The CO linewidths, gas depletion timescale, star formation efficiency, (limits on) dense gas fractions, gas-to-dust ratio, gas mass fraction, SSFR, and stellar mass doubling timescale observed toward HLSW-01 are consistent with "typical" SMGs (e.g., Greve et al. 2005; Gao et al. 2007). The spatial and dynamical structure of the molecular gas reservoir is complex, consistent with what is observed in major mergers in other SMGs (e.g., Tacconi et al. 2008; Carilli et al. 2010; Engel et al. 2010; Bothwell et al. 2010), but higher spatial resolution and a full dynamical lens inversion are required to investigate the velocity structure in more detail (see, e.g., Riechers et al. 2008). The brightest peaks of the $\mathrm{CO}$ emission show a small spatial offset from those observed in the rest-frame optical, suggesting that the most gas-rich, most intensely star-forming regions in HLSW-01 are heavily obscured, consistent with what is observed in unlensed SMGs (e.g., Tacconi et al. 2008; Daddi et al. 2009a, 2009b; Carilli et al. 2010; Riechers et al. 2010).

HLSW-01 is an exceptional example for the level of detail to which the molecular gas properties of "typical" SMGs can be studied with the aid of strong gravitational lensing. Such studies provide crucial insight into the physical processes that accompany the early stellar mass buildup during the most active phases in the evolution of massive galaxies.

We thank the referee for a detailed and helpful report. D.R. acknowledges support from NASA through Hubble Fellowship grant HST-HF-51235.01 awarded by STScI, operated by AURA for NASA, under contract NAS 5-26555. A.J.B. acknowledges support from NSF grant AST-0708653 to Rutgers University. IRAM is supported by INSU/CNRS (France), MPG (Germany), and IGN (Spain). Support for CARMA construction was derived from the Moore and Norris Foundations, the Associates of Caltech, the states of California, Illinois, and Maryland, and the NSF. Ongoing CARMA development and operations are supported by the NSF under a cooperative agreement, and by the CARMA partner universities. The National Radio Astronomy Observatory is a facility of the National Science Foundation operated under cooperative agreement by Associated Universities, Inc.

\section{REFERENCES}

Blain, A. W., et al. 2002, Phys. Rep., 369, 111

Bothwell, M. S., et al. 2010, MNRAS, 405, 219

Carilli, C. L., et al. 2010, ApJ, 714, 1407

Conley, A., et al. 2011, ApJ, 732, L35 (C11)

Daddi, E., et al. 2009a, ApJ, 694, 1517

Daddi, E., et al. 2009b, ApJ, 695, L176

Danielson, A. L. R., et al. 2011, MNRAS, 410, 1687

Downes, D., \& Solomon, P. M. 1998, ApJ, 507, 615

Engel, H., et al. 2010, ApJ, 724, 233

Gao, Y., Carilli, C. L., Solomon, P. M., \& Vanden Bout, P. A. 2007, ApJ, 660, L93

Gao, Y., \& Solomon, P. M. 2004a, ApJS, 152, 63

Gao, Y., \& Solomon, P. M. 2004b, ApJ, 606, 271

Gavazzi, R., et al. 2011, ApJ, submitted (arXiv:1104.4119) (G11)

Greve, T. R., et al. 2005, MNRAS, 359, 1165

Greve, T. R., et al. 2009, ApJ, 692, 1432

Harris, A. I., Baker, A. J., Zonak, S. G., Sharon, C. E., Genzel, R., Rauch, K., Watts, G., \& Creager, R. 2010, ApJ, 723, 1139

Harris, A. I., et al. 2007, in ASP Conf. Ser. 375, From Z-Machines to ALMA: (Sub)Millimeter Spectroscopy of Galaxies, ed. A. J. Baker et al. (San Francisco, CA: ASP), 82

Hughes, D. H., et al. 1998, Nature, 394, 241

Ivison, R. J., et al. 2010a, MNRAS, 404, 198

Ivison, R. J., et al. 2010b, A\&A, 518, L35

Knudsen, K. K., et al. 2007, ApJ, 666, 156

Lagache, G., Puget, J.-L., \& Dole, H. 2005, ARA\&A, 43, 727

Michalowski, M. J., Watson, D., \& Hjorth, J. 2010, ApJ, 712, 942

Oliver, S. J., et al. 2010, A\&A, 518, L21

Ott, M., Witzel, A., Quirrenbach, A., Krichbaum, T. P., Standke, K. J., Schalinski, C. J., \& Hummel, C. A. 1994, A\&A, 284, 331

Renzini, A. 2006, ARA\&A, 44, 141

Riechers, D. A., Walter, F., Carilli, C. L., \& Bertoldi, F. 2007, ApJ, 671, L13

Riechers, D. A., et al. 2008, ApJ, 686, 851

Riechers, D. A., et al. 2010, ApJ, 720, L131

Scott, K., et al. 2011, ApJ, in press (arXiv:1104.4115) (S11)

Smail, I. 2006, arXiv:astro-ph/0603635

Smail, I., Ivison, R. J., \& Blain, A. W. 1997, ApJ, 490, L5

Solomon, P. M., \& Vanden Bout, P. A. 2005, ARA\&A, 43, 677

Spergel, D. N., et al. 2003, ApJS, 148, 175

Spergel, D. N., et al. 2007, ApJS, 170, 377

Swinbank, A. M., et al. 2010, Nature, 464, 733

Tacconi, L. J., et al. 2006, ApJ, 640, 228

Tacconi, L. J., et al. 2008, ApJ, 680, 246 